\title{
Ventilator associated pneumonia in a tertiary care hospital in India: Incidence, etiology, risk factors, role of multidrug resistant pathogens
}

\section{Kalidas Rit, Bipasa Chakraborty, Rajdeep Saha ${ }^{1}$, Udayan Majumder ${ }^{2}$}

Departments of Microbiology, Institute of Post-Graduate Medical Education and Research, Kolkata,

${ }^{1}$ Microbiology, National Medical College, Kolkata, ${ }^{2}$ Department of Anaesthesiology, North Bengal Medical College, Sushrutanagar, Siliguri, West Bengal, India

Address for the Correspondence: Dr. Kalidas Rit, 70B T.C. Mukherjee Street, Rishra, Hooghly - 712 248, West Bengal, India. E-mail: kalidasrit77@gmail.com

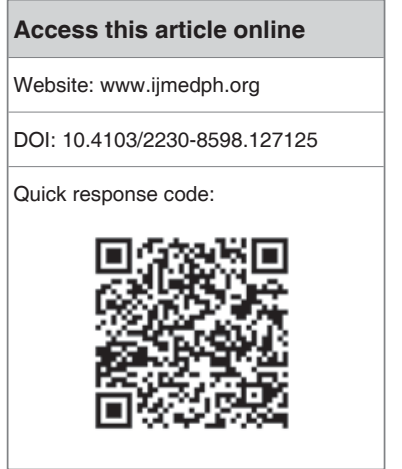

Background: Ventilator associated pneumonia (VAP), a hospital acquired infection (HAl) is seen among critically ill patients on mechanical ventilation (MV) due to various causes, in intensive care units (ICUs). VAP increases morbidity, mortality, as well as the cost of healthcare. Materials and Methods: A prospective study was done over a period of 10 months in a tertiary care hospital in India to determine the incidence, etiological agents, their sensitivity profiles, and risk factors associated with VAP. Combination disc method, ethylenediaminetetraacetic acid (EDTA) disc synergy (EDS) tests, and AmpC disc tests were performed for detection of extendedspectrum beta-lactamases (ESBL), metallo-beta-lactamases (MBL), and AmpC beta-lactamases, respectively. Results: One hundred and forty adult patients, on MV for $48 \mathrm{~h}$ and more, were included and 28 (20\%) developed VAP. The incidence density rate of VAP was 21.875 per 1,000 ventilator days. Most of the patients had late onset VAP $(60.7 \%)$ with average number of days for onset around 8 days. Pseudomonas spp. and Acinetobacter spp. were significantly associated with late onset VAP, whereas Enterobacteriaceae, Staphylococcus aureus, Haemophilus influenzae, Streptococcus pneumoniae, Burkholderia cepacia, and Candida species were commonly isolated from early onset VAP. Polymicrobial infections occurred in 14 cases, so overall 43 VAP pathogens were isolated. Thirty $(69.7 \%)$ of them were multidrug resistant (MDR), among which ESBL contributed 23.25\%, MBL $30.23 \%$, AmpC beta-lactamases $9.30 \%$, and to methicillin resistant $S$. aureus (MRSA) contributed $6.97 \%$. Prior antibiotic therapy $(P<0.0001)$, hospitalization for 5 days or more $(P<0.0001)$, MV for 5 days or more $(P<0.0001)$, supine head position $(P<0.0001)$, reintubation $(P=0.0012)$, and impaired consciousness $(P=0.0191)$ were significant risk factors for VAP. Conclusions: Proper knowledge of risk factors can help identify high risk groups for VAP, among the critically ill patients on MV. MDR pathogens, with production of ESBL, MBL, AmpC beta-lactamases, and MRSA were commonly associated with VAP. So, judicious use of antibiotic is recommended.

Key words: AmpC beta-lactamase, ESBL, metallo-beta lactamase, MRSA, ventilator associated pneumonia

\section{INTRODUCTION}

Ventilator associated pneumonia (VAP) is pneumonia that occurs $48 \mathrm{~h}$ or more after endotracheal intubation and mechanical ventilation (MV) that was not intubating at the time of admission, also including pneumonia developing after extubation. ${ }^{[1]}$ Pneumonia is the second most common intensive care unit (ICU) acquired infection and $86 \%$ of nosocomial pneumonias are VAP. ${ }^{[1]}$ Around $10-20 \%$ of patients on MV for longer than 48 h, develops VAP. ${ }^{[2,3]}$ Early onset VAP, which occurs during the first 4 days of MV is usually less severe, associated with a better prognosis and more likely caused by antibiotic sensitive bacteria. Late onset VAP, which develops 5 or more days after initiation of $\mathrm{MV}$, is caused by multidrug resistant (MDR) pathogens and associated with increased mortality and morbidity. ${ }^{\left[{ }^{4}\right]}$ The common pathogens causing VAP include aerobic gram negative rods such as Pseudomonas aeruginosa, Acinetobacter species, Klebsiella pneumoniae, and Escherichia coli. ${ }^{[1,5,6]}$ VAP due to methicillin resistant Staphylococcus aureus (MRSA) has been rapidly emerging. ${ }^{[5,6]}$ Treatment of VAP is usually supportive, along with administration of proper antibiotic. The selection of proper antimicrobial agents, active against the VAP pathogens is an important determinant for reducing morbidity and 
mortality. Appropriate antimicrobial therapy, when initiated early, has shown to reduce mortality among critically ill patients with VAP. Late onset VAP is commonly associated with administration of inappropriate antibiotics and caused by MDR pathogens like Pseudomonas species and Acinetobacter species. MDR is due to production of extended-spectrum beta-lactamases (ESBL), AmpCbeta lactamase, and MBL. ${ }^{[5,6]}$ The etiological agents of VAP vary with different patient population and types of ICU. Therefore, the local microbial flora causing VAP needs to be studied in each setting to guide rational utilization of antimicrobial agents.

\section{MATERIALS AND METHODS}

This prospective study was conducted in two ICU of a tertiary care hospital in Kolkata from May 2011 to February 2012. Necessary clearance from institutional ethical committee was obtained prior to the study. Patients were excluded if they were intubated for less than $48 \mathrm{~h}$ or if the reason for intubation was suspected to be hospital acquired pneumonia. The diagnosis of VAP was based on clinical and microbiological criteria. During 10 months study period, 964 patients were admitted in two ICUs and 222 patients were intubated and put on MV. One hundred and forty-six patients received MV for more than $48 \mathrm{~h}$ in the ICU, of which six developed pneumonia within 48 $\mathrm{h}$, and were excluded from the study. So 140 patients on MV were included in this study and prospectively reviewed. All these patients included were monitored at 2 days interval for development of VAP using clinical and microbiological criteria until either discharge or death. Details of antibiotic therapy, use of steroids, duration of hospitalization and MV, position of patient, use of sedatives, presence of neurological disorder, impaired consciousness, and other important parameters studied are summarized in Table 1. Any patient with modified Clinical Pulmonary Infection Score (CPIS) $>6$ (a clinical score of 0-12 based on the six variables like body temperature, leukocyte count, volume and character of tracheal secretion, arterial oxygenation, chest radiograph findings, gram stain results, and results of culture of tracheal aspirate specimen $)^{[1,7]}$ and quantitative culture of endotracheal aspirate with growth thresholds greater than equal to $10^{6} \mathrm{cfu} / \mathrm{ml}^{[8]}$ was taken as a case of VAP. Based on these criteria, 28 of 140 patients were diagnosed with VAP. The organisms isolated by quantitative culture of the endotracheal aspirate (EA) from VAP patients were identified based on standard microbiological technique. ${ }^{[9]}$ The antibiotic susceptibility test was done by Modified Kirby Bauer Disc diffusion method. ${ }^{[10]}$ Routinely used antibiotics as shown in Tables 2 and 3 were tested for susceptibility of Enterobacteriaceae, P. aeruginosa, Acinetobacter spp, S. aureus, Burkholderia cepacia, Streptococcus pneumoniae, and Haemophilus influenzae. Susceptibility of $S$. aureus to oxacillin was determined using oxacillin-salt-screen-agar containing $6 \mu \mathrm{g} / \mathrm{ml}$ oxacillin and $4 \%$ $\mathrm{NaCl} \cdot{ }^{[11]}$ Combination disc method using ceftazidime alone and in combination with clavulanic acid was performed for detection of ESBL among the members of Enterobacteriaceae. ${ }^{[12]}$ Increase of $\geq 5 \mathrm{~mm}$ in zone of inhibition for ceftazidime-clavulanic acid disc compared to the ceftazidime disc alone was taken as confirmatory evidence of ESBL production. AmpC disc test was performed for detection of AmpC-beta lactamase. A flattening or indentation of the cefoxitin inhibition zone in the vicinity of the disc with test strains was interpreted as positive. ${ }^{[13]}$ Ethylenediaminetetraacetic acid (EDTA) disc synergy test was done using imipenem for detection of metallo-beta-lactamase with the imipenem resistant strains. ${ }^{[14]}$ Two imipenem discs were placed on the surface of agar plate at distance of $25 \mathrm{~mm}$ and $750 \mu \mathrm{g}$ of EDTA was added to one of them. After 24 hours of incubation at $37^{\circ} \mathrm{C}$ the inhibition zones of imipenem and imipenem-EDTA disc were compared. If the increase in inhibition zone with the imipenem-EDTA disc was $\geq 7 \mathrm{~mm}$ than the imipenem alone, it was considered MBL positive. Among the gram negative bacilli (GNB) isolated from VAP, those producing ESBL, AmpC beta-lactamases or MBL, and resistant to three or more antimicrobial classes were defined as MDR pathogens. Acinetobacter baumanii was classified as MDR if resistant to four or more antibiotic classes. Among the gram positive cocci, MRSA and S. pneumoniae, resistant to penicillin and at least two other antibiotic classes were defined as MDR pathogens. ${ }^{[15]}$

\section{RESULTS}

Out of the 140 selected patients $28(20 \%)$ of them were diagnosed with VAP with CPIS $>6$ and fulfilling microbiological criteria. $67.85 \%(19 / 28)$ of the patients were males with mean age of 60.8 years. The incidence-density rate of VAP was 21.875 per 1,000 ventilator days.

Administration of prior antibiotic therapy, hospitalization for 5 days or more, MV for 5 days or more, reintubation, impaired consciousness, supine head position, and comorbid condition like hypertension, diabetes mellitus were significant risk factors associated with VAP by chi-square (Fisher's exact) test, as shown in Table 1.

$60.7 \%(17 / 28)$ of the patients had late onset VAP with average number of days for onset around 8 days. Polymicrobial infections occurred in 50\% (14/28) of cases, so overall 43 VAP pathogens were isolated, of which 17 pathogens were from early onset VAP and 26 from late onset VAP. The most common causative agents of early onset VAP were members of Enterobacteriaceae $(35.2 \%$ or $6 / 17$ ) and Acinetobacter spp (17.6\% or 3/17). Methicillin sensitive $S$. aureus (MSSA), were the most common gram positive bacteria $(11.7 \%$ or $2 / 17$ ) associated with early onset VAP, whereas in late onset VAP, MRSA $(7.6 \%$ or $2 / 26)$ were more commonly isolated than MSSA (3.8\% or $1 / 26)$. Acinetobacter spp (34.6\% or $9 / 26)$ and P. aeruginosa $(30.7 \%$ or $8 / 26)$ were the most common pathogens causing late onset VAP, whereas Enterobacteriaceae, H. influenzae, S. aureus, S. pneumoniae, and Candida spp were more common in early onset VAP. The antibiotic resistance profile of the isolated pathogens of early onset VAP and late onset VAP are summarized in Tables 2 and 3.

Drug resistance has been found in $69.76 \%(30, n=43)$ of the nosocomial isolates, among which ESBL contributed $23.25 \%(10, n=43)$, MBL $30.23 \%(13, n=43)$, Amp C betalactamases $9.30 \%(4, n=43)$, and MRSA contributed $6.97 \%$ 


\begin{tabular}{|c|c|c|c|c|c|}
\hline Risk factors & Non-VAP $(n=112)$ & $\operatorname{VAP}(n=28)$ & Relative risk & 95\% confidence interval & $P$-value \\
\hline Hospitalization for 5 days or more & 43 & 28 & 0.6056 & $0.5020-0.7307$ & $<0.0001$ \\
\hline Mechanical ventilation for 5 days or more & 10 & 17 & 0.4103 & $0.2500-0.6735$ & $<0.0001$ \\
\hline Emergency intubation & 94 & 20 & 1.191 & $0.9093-1.560$ & 0.1721 \\
\hline Reintubation & 1 & 5 & 0.2012 & $0.0335-1.207$ & 0.0012 \\
\hline Intravenous sedatives & 66 & 18 & 0.9565 & $0.8106-1.129$ & 0.6704 \\
\hline Prior antibiotic therapy & 41 & 26 & 0.6292 & $0.5179-0.7643$ & $<0.0001$ \\
\hline Impaired consciousness & 40 & 17 & 0.8090 & $0.6696-0.9773$ & 0.0191 \\
\hline Tracheostomy & 12 & 4 & 0.9300 & $0.6918-1.250$ & 0.5269 \\
\hline Steroid therapy & 18 & 6 & 0.9255 & $0.7228-1.185$ & 0.5754 \\
\hline Supine head position & 22 & 18 & 0.6111 & $0.4582-0.8150$ & $<0.0001$ \\
\hline Surgical causes & 16 & 4 & 1.000 & $0.7892-1.267$ & 1.0000 \\
\hline Neurological disorders & 40 & 12 & 0.9402 & $0.7864-1.124$ & 0.5167 \\
\hline
\end{tabular}

\section{Table 2: Susceptibility profile of the etiological agents of early onset VAP}

\begin{tabular}{|c|c|c|c|c|c|c|c|c|c|c|c|c|}
\hline \multirow{2}{*}{$\begin{array}{l}\text { Etiological agents } \\
\text { (no. of isolates) }\end{array}$} & \multicolumn{12}{|c|}{ Antibiotic resistant pattern (\%) } \\
\hline & AMP & TIC & AMK & CIP & CAZ & $\mathrm{CX}$ & AT & CPM & CFS & PTZ & IMP & CL \\
\hline Acinetobacter baumanii (2) & - & 100 & 100 & 100 & 100 & 50 & 50 & 100 & - & 50 & 50 & 0 \\
\hline A. Iwoffii (1) & - & 100 & 100 & 100 & 100 & 100 & 0 & 100 & - & 0 & 100 & 0 \\
\hline Pseudomonas aeruginosa (1) & - & 100 & 100 & 0 & 100 & 0 & 100 & 50 & - & 0 & 0 & 0 \\
\hline Burkholderia cepacia (1) & - & 100 & 100 & 100 & 100 & 100 & 0 & 100 & - & 100 & 100 & 0 \\
\hline Klebsiella pneumoniae (2) & 100 & - & 50 & 50 & 50 & 50 & 50 & 50 & 50 & - & 0 & - \\
\hline Escherichia coli (1) & 100 & - & 0 & 0 & 0 & 0 & 0 & 0 & 0 & - & 0 & - \\
\hline Citrobacter koseri (1) & - & 100 & 0 & 100 & 100 & 100 & 100 & 0 & 100 & - & 0 & - \\
\hline Enterobacter spp (1) & - & 100 & 0 & 0 & 100 & 100 & 100 & 100 & - & 100 & 0 & 0 \\
\hline \multirow[t]{2}{*}{ Serratia marcescens (1) } & - & 100 & 100 & 0 & 100 & 100 & 100 & 100 & - & 0 & 0 & 0 \\
\hline & AMP & $A Z$ & CTR & CPM & TET & CIP & & & PTZ & & & \\
\hline \multirow[t]{2}{*}{ Haemophilus influenzae (1) } & 0 & 0 & 0 & 0 & 0 & 0 & & & 0 & & & \\
\hline & AMP & $\mathrm{AZ}$ & CAZ & OXA & TET & CIP & & & AMK & $\mathrm{LZ}$ & VAN & \\
\hline MSSA (2) & 50 & 0 & 50 & 0 & 0 & 0 & & 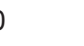 & 0 & 0 & 0 & \\
\hline \multirow[t]{2}{*}{ MRSA (1) } & 100 & 100 & 100 & 100 & 0 & 0 & & 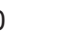 & 0 & 0 & 0 & \\
\hline & PEN & $\mathrm{AZ}$ & CTR & AMC & TET & CIP & & DT & IMP & $\mathrm{LZ}$ & VAN & \\
\hline Streptococcus pneumoniae (1) & 100 & 100 & 0 & 0 & 0 & 0 & & 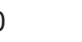 & 0 & 0 & 0 & \\
\hline Candida spp (1) & - & - & - & - & - & - & & - & & - & - & \\
\hline
\end{tabular}

VAP = Ventilator associated pneumonia, AMP = Ampicillin, TIC = Ticarcillin, AMK = Amikacin, CIP = Ciprofloxacin, CAZ = Ceftazidime, CX = Cefoxitin, AT = Aztreonam, $\mathrm{CPM}=$ Cefepime, $\mathrm{CFS}=$ Cefoperazone/sulbactum, PTZ = Piperacillin/tazobactum, IMP = Imipenem, $C L=$ Colistin, $\mathrm{AZ}=\mathrm{Azithromycin}, \mathrm{CTR}=\mathrm{Ceftriaxone}, \mathrm{OXA}=\mathrm{Oxacillin}$, TET = Tetracycline, COT = Cotrimoxazole, LZ = Linezolid, VAN = Vancomycin, PEN = Penicillin, AMC = Amoxicillin-clavulanic acid, MSSA = Methicillin sensitive Staphylococcus aureus, MRSA = Methicillin resistant Staphylococcus aureus

\begin{tabular}{|c|c|c|c|c|c|c|c|c|c|c|c|c|}
\hline \multirow{2}{*}{$\begin{array}{l}\text { Etiological agents } \\
\text { (no. of isolates) }\end{array}$} & \multicolumn{12}{|c|}{ Antibiotic resistant pattern (\%) } \\
\hline & AMP & TIC & AMK & CIP & CAZ & CX & AT & CPM & CFS & PTZ & IMP & $\mathrm{CL}$ \\
\hline Acinetobacter baumanii (6) & - & 83.3 & 33.3 & 50 & 100 & 33.3 & 50 & 33.3 & - & 33.3 & 50 & 33.3 \\
\hline A. Iwoffii (3) & - & 100 & 66.6 & 66.6 & 100 & 33.3 & 33.3 & 33.3 & - & 66.6 & 33.3 & 0 \\
\hline Pseudomonas aeruginosa (8) & - & 75 & 62.5 & 50 & 75 & 62.5 & 25 & 25 & - & 50 & 50 & 0 \\
\hline Escherichia coli (2) & 100 & - & 50 & 50 & 100 & 50 & 50 & 50 & 50 & 0 & 50 & - \\
\hline Enterobacter spp (1) & - & 100 & 0 & 100 & 100 & 100 & 100 & 100 & - & 100 & 0 & 0 \\
\hline Klebsiella pneumoniae (2) & 100 & - & 100 & 100 & 100 & 50 & 50 & 100 & 100 & - & 50 & 0 \\
\hline \multirow[t]{2}{*}{ Proteus mirabilis (1) } & 100 & - & 0 & 0 & 100 & 0 & 0 & 0 & 0 & - & 0 & - \\
\hline & AMP & $A Z$ & CAZ & OXA & TET & CIP & COT & AMK & $\mathrm{LZ}$ & VAN & & \\
\hline MSSA (1) & 100 & 0 & 100 & 0 & 0 & 0 & 0 & 0 & 0 & 0 & & \\
\hline MRSA (2) & 100 & 50 & 50 & 100 & 50 & 50 & 50 & 50 & 0 & 0 & & \\
\hline
\end{tabular}

VAP = Ventilator associated pneumonia, AMP = Ampicillin, TIC = Ticarcillin, AMK = Amikacin, CIP = Ciprofloxacin, CAZ = Ceftazidime, CX = Cefoxitin, AT = Aztreonam, $\mathrm{CPM}=$ Cefepime, $\mathrm{CFS}=$ Cefoperazone/sulbactum, PTZ = Piperacillin/tazobactum, IMP = Imipenem, CL = Colistin, AZ = Azithromycin, CTR = Ceftriaxone, OXA = Oxacillin, TET = Tetracycline, COT = Cotrimoxazole, LZ = Linezolid, VAN = Vancomycin, PEN = Penicillin, AMC = Amoxicillin-clavulanic acid, MSSA = Methicillin sensitive Staphylococcus aureus, MRSA = Methicillin resistant Staphylococcus aureus 
$(3, n=43)$ [Figure 1]. ESBL was produced by 33.3\% (4/12) of Acinetobacter spp, 33.3\% (3/9) of P. aeruginosa, and 25\% (3/12) of Enterobacteriaceae isolated from VAP patients. MBL was produced mainly by nonfermenters, $P$. aeruginosa $(44.4 \%$ or 4/9) and Acinetobacter spp (50\% or 6/12). Also 16.66\% (2/12) Enterobacteriaceae produced MBL. AmpC beta-lactamases were produced by $33.33 \%(4 / 12)$ of Enterobacteriaceae [Figure 2]. Among the $S$. aureus isolates, 50\% were methicillin resistant. Majority of nonfermenter like Pseudomonas, Acinetobacter spp were resistant to ticarcilin, ceftazidime, and amikacin.

\section{DISCUSSION}

VAP is an important nosocomial infection among the critically ill patients, receiving MV. VAP are significant public health issues with high morbidity and mortality and increased costs of treatment. The incidence-density rate of VAP in our study was 21.875 per 1,000 ventilator days. And data from Asian countries suggest an incidence-density rate of VAP varying from 3.5 to 46 per 1,000

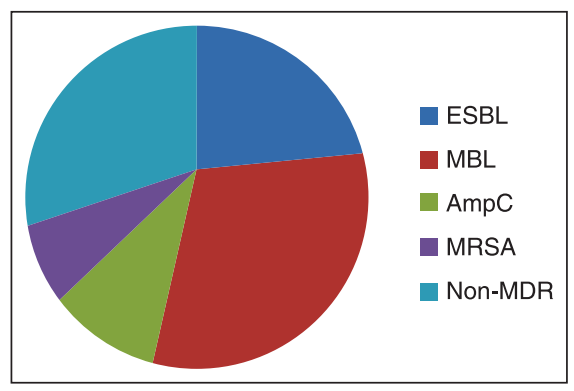

Figure 1: Comparison between different patterns of multidrug resistant (MDR) pathogens and non-MDR pathogens isolated from ventilator associated pneumonia (VAP) patients ventilator days. ${ }^{[5,8]}$ Our study showed a preponderance of male sex $(67.85 \%$ or $19 / 28)$ for VAP.

Similar to other studies, ${ }^{[8]}$ in our study also, Acinetobacter spp were found to be the most common pathogen in VAP $(27.90 \%$ or $12 / 43)$, followed by $P$. aeruginosa $(20.93 \%$ or $9 / 43)$, S aureus $(13.95 \%$ or $6 / 43$, of which $50 \%$ were MRSA), Klebsiella pneumoniae $(9.3 \%$ or $4 / 43)$, and E. coli $(6.97 \%$ or $3 / 43)$. In patients with late-onset VAP, most common organism isolated were the nonfermenters like Acinetobacter spp and $P$. aeruginosa. Even in patient with early onset VAP, Acinetobacter spp was the most common pathogen isolated, and most of them are MDR pathogens. Enterobacteriaceae like E. coli, K. pneumoniae, Serratia marcescens, Citrobacter spp, Enterobacter spp, B. cepacia, S. aureus, H. influenzae, S. pneumoniae, and Candida species were also responsible for good number of cases particularly in early onset VAP. These findings are well-corroborated with the findings of other studies conducted elsewhere in India. ${ }^{[3,5]}$

Regarding the susceptibility profiles of the etiological agents of early onset VAP colistin was found to be most effective antibiotic followed by piperacillin/tazobactum combination and the imipenem. Ampicillin and ticarcillin were least effective drugs. Both MSSA and MRSA strains showed $100 \%$ susceptibility to vancomycin. Regarding the susceptibility profile of the etiological agents of late onset VAP colistin remain the drug of choice against all isolated GNB except A. baumanii which showed 33\% resistance. Piperacillin/tazobactum combination and the imipenem remain the second most ones.

As the incidence of MDR-pathogens was quite high (69.76\%) in our study, this indicates that need for appropriate empirical treatment of VAP with proper antibiotics, effective against these MDR pathogens are required. ${ }^{[16]}$ Production of various forms of beta-lactamases

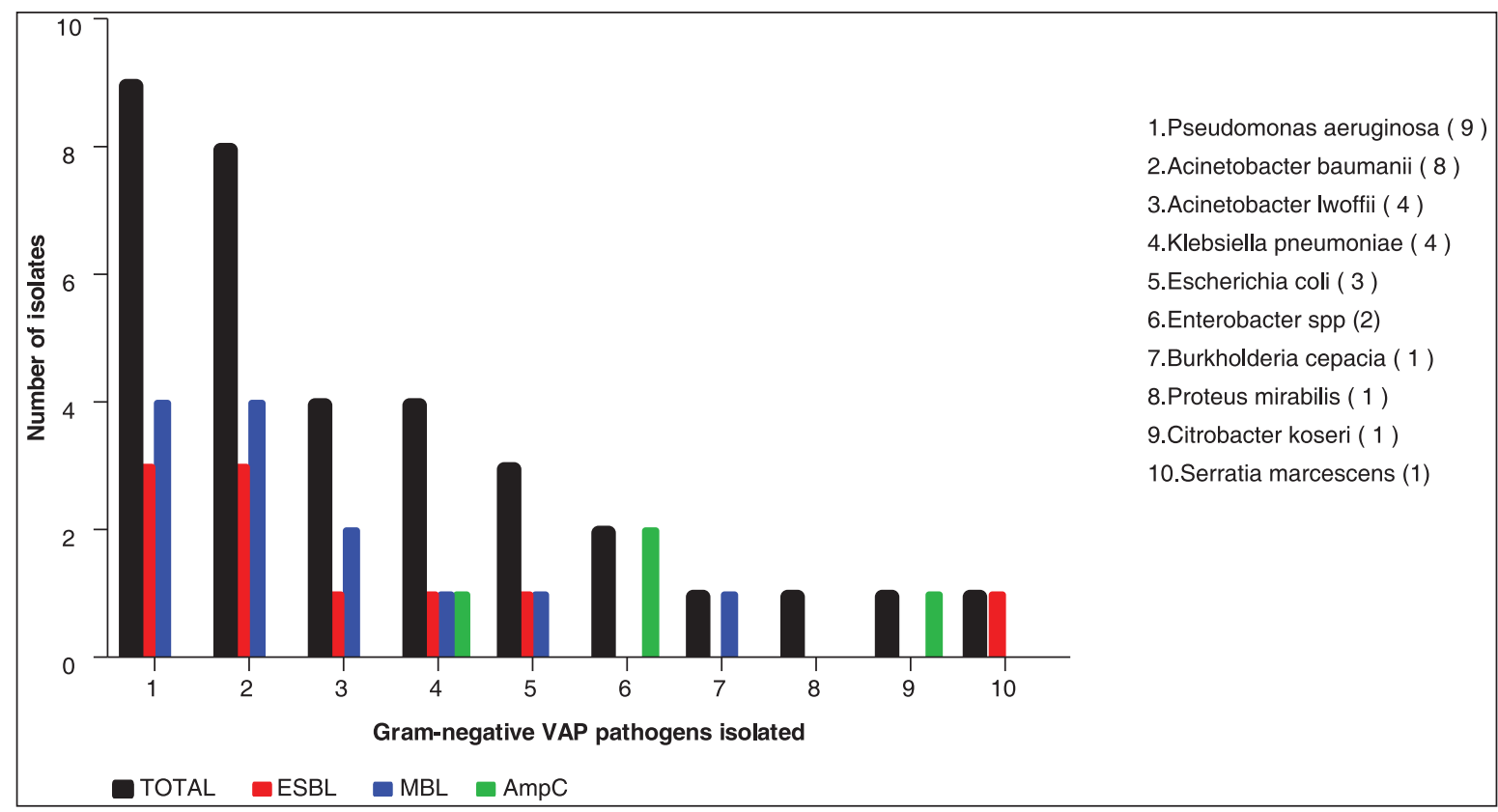

Figure 2: Production of extended-spectrum beta-lactamase (ESBL), metallo-beta-lactamases (MBL), and AmpC beta-lactamases enzymes among the VAP pathogens 
like ESBL, MBL, and AmpC beta-lactamase were responsible for this MDR. Similar to other studies, ${ }^{[17,18]}$ ESBL and and MBL were produced by most of the nonfermenter. AmpC beta-lactamases were mainly produced by members of Enterobacteriaceae like Citrobacter spp, Enterobacter spp, and K. pneumonia, but not by nonfermenters in our study. Late onset VAP was more commonly associated with MDR pathogens $(76.9 \%$ or $20 / 26)$ compared to early onset VAP $(58.8 \%$ or $10 / 17)$. According to our study, colistin was highly effective against Acinetobacter spp, though few $A$. baumanii infections showed resistance to colistin, while piperacillintazobactum combination had good activity against $P$. aeruginosa. So these two drugs showed good in vitro activity against MDR nonfermenters.

In both early and late onset VAP GNB were more common isolates in comparison with gram positive one. Among the isolated GNB's, a significant number of VAP cases were due to nonfermenter which were often MDR strains.

In the present study we found that, prior antibiotic therapy, hospitalization of 5 days or more, MV for 5 days and more, and supine head position were the most significant risk factors associated with $\operatorname{VAP}(P<0.0001)$. Other significant risk factors were impaired consciousness $(P=0.0191)$ and reintubation $(P=0.0012)$. Analysis of these risk factors were done by chi-square (Fisher's exact) test. These findings are similar to other studies also. ${ }^{[19,20]}$

This emphasizes the need for judicious selection and rational use of appropriate antibiotic which may reduce patient colonization and subsequent VAP by MDR pathogens. Similarly, unnecessary prolonged hospitalization and prolonged intubation and MV of the patient should be avoided as far as possible, rather noninvasive techniques for ventilation should be tried whenever possible. But it may not be possible in most situations. However, the knowledge of these risk factors can help identify high risk groups for VAP, and can suggest the possibility of infection due to MDR pathogens especially in late onset VAP. On the basis of antibiotic susceptibility tests of our study and similar other studies, ${ }^{[1]}$ empirical therapy can be broadened to include either an antipseudomonal cephalosporin (cefepime or ceftazadime), a carbepenem (imipenem or meropenem), or a $\beta$-lactam $/ \beta$-lactamase inhibitor (pipercacillin-tazobactam) plus an antipseudomonal fluoroquinolone (ciprofloxacin or levofloxacin), or an aminoglycoside (amikacin, gentamicin, or tobramycin) plus linezolid or vancomycin. Patients with impaired consciousness, inadequate cough reflexes and supine head position are predisposed to aspiration and for developing VAP.

Our study was conducted in a resource-limited setting with only small number of patients with VAP in a single center which could be considered a limitation of our study. Also we recognize that the findings of this study may not necessarily reflect the situations in other similar centers in our region. In addition, acute physiology and chronic health evaluation scores which would have been helpful in assessing the severity of illness were not calculated.
To conclude, awareness of independent risk factors documented in this study may assist in identifying patients at higher risk for VAP and help in implementing appropriate preventive measures, including proper positioning and patient care and modulating intervention measures during management. Limitations of this study were small sample size; inadequate determination of risk factors of development of VAP in predisposed person. Also knowledge of the susceptibility pattern of the local pathogens should guide the choice of antibiotics, in addition to the likelihood of organisms, as there is an increasing prevalence of MDR pathogens in late onset VAP.

\section{ACKNOWLEDGMENT}

Dr Prasanta K Maiti, MD, Professor and Head of the department, Department of Microbiology, Institute of Post-Graduate Medical Education \& Research 244 AJC Bose Road. Kolkata-700020 prasantamaiti123@ rediffmail.com for supporting our research work.

\section{REFERENCES}

1. Koenig SM, Truwit JD. Ventilator-associated pneumonia: Diagnosis, treatment, and prevention. Clin Microbiol Rev 2006;19:637-57.

2. Morehead RS, Pinto SJ. Ventilator-associated pneumonia. Arch Intern Med 2000;160:1926-36.

3. Joseph NM, Sistla S, Dutta TK, Badhe AS, Parija SC. Ventilatorassociated pneumonia in a tertiary care hospital in India: Incidence and risk factors. J Infect Dev Ctries 2009;3:771-7.

4. American Thoracic Society, Infectious Diseases Society of America. Guidelines for the management of adults with hospital-acquired, ventilatorassociated, and healthcare-associated pneumonia. Am J Respir Crit Care Med 2005; 171:388-416.

5. Rakshit P, Nagar VS, Deshpande AK. Incidence, clinical outcome, and risk stratification of ventilator-associated pneumonia-a prospective cohort study. Indian J Crit Care Med 2005;9:211-6.

6. Joseph NM, Sistla S, Dutta TK, Badhe AS, Rasitha D, Parija SC. Ventilator associated pneumonia in a tertiary care hospital in India: Role of multidrug resistant pathogens. J Infect Dev Ctries 2010;4:218-25.

7. Fartoukh M, Maitre B, Honore S, Cerf C, Zahar JR, Brun-Buisson C. Diagnosing pneumonia during mechanical ventilation: The clinical pulmonary infection score revisited. Am J Respir Crit Care Med 2003;168:173-9.

8. Chawla R. Epidemiology, etiology, and diagnosis of hospital-acquired pneumonia and ventilator-associated pneumonia in Asian countries. Am J Infect Control 2008;36(4 Suppl):S93-100.

9. Collee JG, Marmion BP, Fraser AG, Simmons A. Mackie and Mccartney's Practical Medical Microbiology. $14^{\text {th }}$ ed. New York: Churchill Livingstone; 1996. p. 978.

10. Clinical Laboratory Standards Institute. 2006. Performance standards for antimicrobial disk susceptibility tests; Approved standard-9 th $^{\text {th }}$ ed. CLSI document M2-A9. 26:1. Clinical Laboratory Standards Institute. Wayne.

11. Clinical Laboratory Standards Institute. 2005. Methods for dilution antimicrobial susceptibility tests for bacteria that grow aerobically. Approved standard, $6^{\text {th }}$ ed. CLSI document M7-A6. CLSI. Wayne.

12. Thomson KS, Sanders CC. Detection of extended-spectrum betalactamases in members of the family Enterobacteriaceae: Comparison of the double-disk and three-dimensional tests. Antimicrob Agents Chemother 1992;36:1877-82.

13. Singhal S, Mathur T, Khan S, Upadhyay DJ, Chugh S, Gaind R, et al. Evaluation of methods for AmpC beta-lactamase in gram negative clinical isolates from tertiary care hospitals. Indian J Med Microbiol 2005;23:120-4.

14. Yong D, Lee K, Yum JH, Shin HB, Rossolini GM, Chong Y. Imipenem EDTA disk method for differentiation of Metallobetalactamase producing clinical isolates of Pseudomonas spp. and Acinetobacter spp. J Clin Microbiol 2002;40:3798-801. 
15. Balakrishnan I. Streptococcus pneumoniae. In: Gillespie SH, Hawkey PM, editors. Principles and Practice of Clinical Bacteriology. $2^{\text {nd }}$ ed. West Sussex: John Wiley \& Sons Ltd; 2006. p. 41-57.

16. Erbay $\mathrm{RH}$, Yalcin AN, Zencir M, Serin S, Atalay H. Costs and risk factors for ventilator-associated pneumonia in a Turkish university hospital's intensive care unit: A case-control study. BMC Pulm Med 2004;4:3.

17. Lee K, Lee WG, Uh Y, Ha GY, Cho J, Chong Y. VIM- and IMP-type metallo-beta-lactamase-producing Pseudomonas spp. and Acinetobacter spp. in Korean hospitals. Emerg Infect Dis 2003;9:868-71.

18. Quale J, Bratu S, Landman D, Heddurshetti R. Molecular epidemiology and mechanisms of carbapenem resistance in Acinetobacter baumannii endemic in New York city. Clin Infect Dis 2003;37:214-20.
19. Hanson LC, Weber DJ, Rutala WA. Risk factors for nosocomial pneumonia in the elderly. Am J Med 1992;92:161-6.

20. Celis R, Torres A, Gatell JM, Almela M, Rodriguez-Roisin R, Agusti-Vidal A. Nosocomial pneumonia. A multivariate analysis of risk and prognosis. Chest 1988;93:318-24.

How to cite this article: Rit K, Chakraborty B, Saha R, Majumder $U$. Ventilator associated pneumonia in a tertiary care hospital in India: Incidence, etiology, risk factors, role of multidrug resistant pathogens. Int J Med Public Health 2014;4:51-6.

Source of Support: Nil, Conflict of Interest: None declared. 